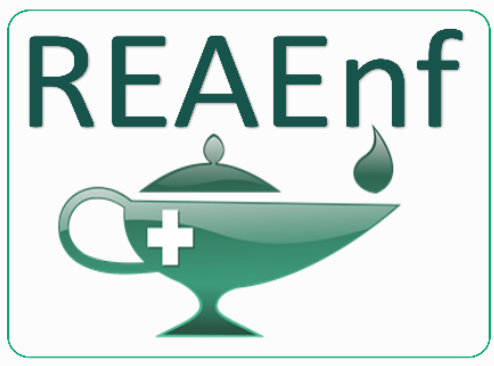

Revista Eletrônica Acervo Enfermagem

\section{REVISÃO BIBLIOGRÁFICA}

Recebido em: 10/2019

Aceito em: $11 / 2019$

Publicado em: 2/2020

\title{
O papel do enfermeiro na prevenção do desmame precoce
}

\author{
The role of the nurse in preventing early weaning \\ El papel del enfermero en la prevención suspensión prematura del amamantamiento
}

Andréia Andrade dos Santos ${ }^{1 *}$, Márcio Antônio Resende ${ }^{1}$ Gabriela Pinto Maia ${ }^{1}$, Nayara Cristina de Jesus Carvalho ${ }^{1}$, Aristarco de Pinho Ferreira Júnior ${ }^{2}$.

Resumo: desmame precoce é um desafio mundial e os profissionais de saúde tem um papel importante no apoio a amamentação exclusiva. O objetivo deste trabalho é identificar os fatores relacionados ao desmame precoce e o papel do enfermeiro na promoção do aleitamento materno, da assistência no pré-natal e na puericultura da atenção básica à área hospitalar; pré-parto, parto e puerpério.Trata-se de um estudo descritivo a partir de uma revisão bibliográfica crítica, realizada por meio de busca em base de dados de artigos científicos. $O$ estudo permitiu compreender que a prática do aleitamento materno traz benefícios tanto para a mãe, quanto para o bebê, além de reforçar o vínculo entre eles. Sabendo que o desmame precoce ocorre por diversos fatores como: a idade materna, primariedade, baixo nível de escolaridade, uso precoce de fórmulas lácteas e chupetas, patologias relacionadas às mamas, trabalho materno, urbanização, tabagismo, falta de incentivo da família e da sociedade, além de deficiências na atenção à saúde, cabe ao enfermeiro garantir a continuidade do aleitamento materno através de educação em saúde no pré-natal, parto e no puerpério, principalmente nos primeiros dias após o parto além de envolver a família nesse momento.

Palavras-chave: Aleitamento materno, Desmame, Cuidados de enfermagem.

\begin{abstract}
The early weaning is a world's (or global) chalenge and healthcare's professionals have an importante role in supporting exclusive breastfeeding. The aim of this paper is to identify the factors related to the early weaning and the nurse's role in promoting breastfeeding, since assistance in the prenatal and in the childcare of the primary care to hospital area; antipartum, childbirth and postpartum. It's about a descriptive study from a critical bibliographic review, performed by searching in database of scientific articles. The study allowed to understand that the practice of breastfeeding brings benefits to both mother and baby, besides reinforcing the bond between them. Knowing that the early weaning takes place for several reasons, such as maternal age, firstness, low education level, premature use of milk formulas and pacifiers breast-related disorders, maternal labor, urbanization, smoking, lack of incentive from family and society, besides deficiencies in the attention to health, it is the nurse's responsibility to ensure the continuity of breastfeeding through health education in prenatal, childbirth and in the postpartum period, especially in the first days after delivery, as well as to involve the family in this moment.
\end{abstract}

Keywords: Breast feeding, Weaning, Nursing care.

${ }^{1}$ Centro Universitário Presidente Tancredo de Almeida Neves (UNIPTAN), São João del-Rei-MG.

*E-mail: andreia.santos@uniptan.edu.br

2 Universidade Federal de São João del-Rei (UFSJ), São João del-Rei-MG. 
Resumen: El destete temprano es un desafío mundial (o global) y los profesionales del cuidado de la salud tienen un rol importante en apoyar la lactancia materna exclusiva. El objetivo de este estudio es identificar los factores relacionados con el destete temprano y el rol del enfermero en promover la lactancia materna, desde la asistencia en el prenatal y el cuidado de niños de primer cuidado hasta el área hospital; antipartum, parto y postpartum. Es sobre un estudio descriptivo de una revisión bibliográfica crítica, realizado buscando en una base de datos de artículos científicos. El estudio permitió entender que la práctica de la lactancia materna trae beneficios a ambos, madre y bebe, además de reforzar el vínculo entre ellos. Sabiendo que el destete temprano se lleva a cabo por diferentes razones, como la edad maternal, primeridad, nivel bajo de educación, uso prematuro de fórmulas de leche y pacificadores, trastornos relacionados con los senos, labor maternal, urbanización, fumar, falta de incentivo por parte de la familia y la sociedad, además las deficiencias en la atención a la salud, es la responsabilidad del enfermero asegurar la continuidad de la lactancia materna a través de la educación para la salud en el periodo prenatal, de parto y postpartum. Especialmente en los primeros días después del parto, al igual que involucrar a la familia en este momento.

Palabras clave: Amamantamiento materno, Destete, Cuidados de enfermería.

\section{INTRODUÇÃO}

A prática da amamentação exclusiva é hoje um desafio para a equipe de saúde, o estímulo ao aleitamento materno (AM) incentiva a nutrição, proteção e também o vínculo entre mãe e filho, além de reduzir a mortalidade infantil (ALMEIDA JML, et al., 2015).

Os autores Carvalho MJLN, et al. (2018) e Silva DP, et al. (2017), o AM é uma prática capaz de trazer diversos benefícios para uma criança. O lactente pode se beneficiar em aspectos nutricionais, cognitivos, econômicos, sociais e emocionais. Sabendo disso, a fim de promover e proteger a Saúde da Criança, instituições como a Organização Mundial de Saúde (OMS) e o Ministério da Saúde (MS) do Brasil recomendam a amamentação exclusiva até os seis meses de vida do bebê e complementada por outros alimentos até os dois anos de idade ou mais.

No Brasil, a pesquisa mais atual que analisa a prevalência do AM nas capitais brasileiras e no Distrito Federal foi publicada em 2009. Foi um estudo do MS que teve o objetivo de avaliar a situação da amamentação e da alimentação complementar no país no período de 1999 a 2008, identificando grupos mais vulneráveis e práticas alimentares saudáveis ou não. Foram incluídas 34.366 crianças menores de 1 ano, a partir de representantes das áreas técnicas de saúde da criança das secretarias de saúde, e as análises baseadas nos indicadores propostos pela OMS. Esse estudo demonstra que a prevalência nacional do AM exclusivo em menores de 6 meses foi de $41 \%$, número esse superior a realidade mundial. Entretanto, 0 comportamento desse indicador é bastante heterogêneo, variando de 27,1\% (Cuiabá/MT) até 56,1\% (Belém/PA). A média nacional de duração do AM exclusivo configura a realidade do desmame precoce no país: 54,1 dias (1,8 meses). O desmame precoce é um desafio mundial e o Brasil demonstra estar distante do cumprimento das metas propostas pela OMS e pelo MS (CARVALHO MJLN, et al., 2018).

São necessárias estratégias coletivas para prevenir o desmame precoce que buscam promover hábitos mais saudáveis de alimentação das crianças no primeiro ano de vida. Entretanto, o ato de não amamentar e/ou introduzir outros alimentos precocemente ocorre em $65 \%$ dos casos no mundo, podendo provocar um número expressivo de comorbidades na criança (MONTESCHIO CAC, et al, 2015; OLIVEIRA AK, et al, 2017).

O enfermeiro tem um papel importante no apoio desta prática e deve identificar e oportunizar momentos educativos, para orientar gestantes e puérperas sobre a importância do AM e o manejo da lactação. Atuar junto à população, não somente prestando assistência, mas também na promoção e proteção ao aleitamento materno, que podem ser uma forma mais efetiva de apoio e de incentivo para essas mães no combate ao abandono da amamentação. Tratando de um tema de grande relevância para a saúde pública, faz-se necessário discutir os fatores que levam as mães ao desmame precoce e o papel do enfermeiro na promoção do aleitamento materno (BATISTA KRA, et al., 2013).

REAEnf/EJNC | Vol. 2 | e2232 | DOI: https://doi.org/10.25248/REAenf.e2232.2020 Página 2 de 7 
O objetivo deste trabalho é identificar os fatores relacionados ao desmame precoce e o papel do enfermeiro na promoção do aleitamento materno, da assistência no pré-natal e na puericultura da atenção básica à área hospitalar; pré-parto, parto e puerpério.

\section{REVISÃO BIBLIOGRÁFICA}

O leite materno é um alimento rico em micro e macronutrientes, gorduras, água, que farão com que o bebê cresça saudável, dentro do percentil normal de desenvolvimento. A composição do leite materno se dá por inúmeras substâncias que contribuem na proteção e no desenvolvimento do recém-nascido, o que o torna único e de extrema importância. Ele pode desempenhar diferentes funções como; alimentar, proteger e prevenir de várias doenças; respiratórias, infecciosas, diarreia, autoimunes, entre outras, da infância até a idade adulta (NETO MT, 2006).

Estima-se que o aleitamento materno pode prevenir $72 \%$ das internações infantis causadas por diarreia e $57 \%$ daquelas causadas por infecções respiratórias. Há muitas mortes evitáveis quando o AM não é praticado corretamente, além de contribuições na família e na sociedade (VICTORA CG, et al, 2016; MARQUES ES, et al., 2011).

De acordo com Andrade HS, et al (2018) o leite materno traz também vários benefícios para a mãe, como redução a incidência de câncer de mama, de ovários, além de diminuir o risco de diabetes e fraturas por osteoporose. Na prática da amamentação a involução uterina é mais rápida e pode permitir a eficácia como contraceptivo natural de até $98 \%$ nos seis primeiros meses após o parto e desde que a mãe se mantenha amenorreica. É evidente e bem documentada a contribuição da lactação na Saúde da Mulher. Há ainda estudos que demonstram também menor presença de depleção mineral óssea, osteoporose, fratura patológica e artrite reumatoide. Por fim, existem evidências científicas sobre perda mais rápida de peso na vigência da amamentação exclusiva (REA MF, 2004).

A amamentação está relacionada à alta relação de custo benefício - para a família e para os serviços de saúde - por não depender da aquisição de fórmulas artificiais, além do aumento do vínculo mãe- filho. Atualmente recomenda-se que todos os procedimentos realizados na sala de parto devem procurar propiciar o contato precoce-de preferência pele a pele-entre mãe e bebê, o que irá favorecer o vínculo afetivo e o estabelecimento da prática de amamentar.

O desmame precoce constitui-se o processo no qual se introduz, progressivamente, a alimentação habitual da família para complementar e/ou substituir o leite materno, antes dos seis meses de vida. Segundo a OMS, a Sociedade Brasileira de Pediatria (SBP), e a Organização Pan-Americana da Saúde (OPAS), recomendase o aleitamento materno exclusivo até os seis meses, sem oferecer água ou chá, e introduzir a alimentação complementar após esse período. Sendo assim a criança desmamada deverá receber os alimentos cinco vezes ao dia, diferente das crianças que ainda recebem o leite materno, essas deverão receber três vezes ao dia, intercalando com o aleitamento (SILVA DP, et al., 2017).

A suspensão da amamentação e o desmame precoce podem ser considerados um ato de violência contra a criança, visto que a deixa exposta, correndo risco de adoecer ou morrer por doenças relacionadas à desnutrição. Apenas $35 \%$ das crianças em todo o mundo seguem a recomendação da OMS em relação a nutrição infantil até os dois anos. Ainda que as lactentes recebem incentivos e saibam das vantagens oferecidas pelo aleitamento materno, muitas mulheres não conseguem alcançar a meta, levando o abandono e consequentemente o desmame precoce (JOCA MT, et al., 2005; PEREIRA DE OLIVEIRA AK, et al., 2017).

Neiva FCB, et al (2003) afirma que o aleitamento materno juntamente com o adequado padrão de sucção é a base para a prevenção de alterações fonoaudiológicas no que se refere ao sistema motor-oral. $O$ desmame precoce traz consequências no desenvolvimento motor-oral criança, comprometendo as funções de respiração, mastigação e deglutição. Há também uma associação com outros problemas, como o uso de mamadeiras, provocando má oclusão. O leite humano é capaz de reduzir a mortalidade e a morbidade infantil. Uma criança, menor de 6 meses, que amamenta exclusivamente, possui uma menor probabilidade de apresentar: anemia, diarreias, infecções respiratórias, otites médias, infecções urinárias e doenças alérgicas.

REAEnf/EJNC | Vol. 2 | e2232 | DOI: https://doi.org/10.25248/REAenf.e2232.2020 Página 3 de 7 
Outros autores apontam ainda que crianças desmamadas precocemente demonstram risco 20 vezes maior para a morte por diarreia, infecções respiratórias agudas e outras doenças infecciosas (SILVA WF e GUEDES ZCF, 2013; CARVALHO CF e SILVA MGF, 2005).

Pode-se dizer que o sucesso do AM depende de uma relação multifatorial. Alguns desses fatores relacionam-se a mãe, outros a criança e, ainda, ao ambiente. Entre eles, estão: o trabalho materno, a presença do pai, o nível de escolaridade da mãe, a idade da mãe, as condições do nascimento e o período pós-parto, a influência dos familiares e os hábitos de vida (MOURA ERBB, et al., 2015).

O desmame precoce é um importante problema de saúde pública e pode estar relacionado a várias causas como a idade materna, primariedade, baixo nível de escolaridade, uso precoce de fórmulas lácteas e chupetas, patologias relacionadas às mamas, trabalho materno, urbanização, tabagismo, falta de incentivo da família e da sociedade, além de deficiências na atenção à saúde. Os motivos podem estar ligados à cultura, estilo de vida e influência da sociedade (CIAMPO LAD, et al., 2006).

Há ainda mitos e crenças como possíveis causas de desmame precoce: sendo elas: "Meu leite é fraco", "pouco leite", "o bebê não quis pegar o peito", "o leite materno não mata a sede do bebê" e "os seios caem com a lactação" são relatos recorrentes de mães que evidenciam a insegurança da mulher ante questões do cotidiano materno durante a amamentação. Dessa forma, o enfermeiro deve atuar de forma imprescindível repassando informações e transmitindo segurança para a mãe, afim de garantir o sucesso no aleitamento materno. E essa insegurança da mãe em relação a concepção de leite fraco, insuficiente e que não sustenta a criança são fatores que ajudam na diminuição do aleitamento materno e que são alimentados por empresas de alimentos artificiais que lançam a cada dia propagandas que ajuda quebrar essa confiança da mãe em relação a sua capacidade de amamentar (MARQUES ES, et al., 2011).

Um dos problemas no puerpério, que pode levar ao desmame precoce, são as patologias relacionadas as mamas como dor durante a amamentação, ingurgitamento mamário, mastite, sucção em má posição, mamilos planos e invertidos, abscessos mamários. Assim sendo, é necessário a caracterização clínica precoce desses fatores para que sejam realizadas medidas preventivas, favorecendo a redução dos casos novos. Já as mamas ingurgitadas possuem características, como: quentes, dolorosas, pesadas e edemaciadas, "empedradas", dificuldade de o leite fluir, podendo ocorrer mal-estar geral, cefaleia, febre e calafrios (SALES NA, et al, 2000; SANTOS EKA, 2005; TAMEZ RN e SILVA MJP, 2006).

Além dos fatores ligados a falta de informação está o retorno ao trabalho, onde ocasiona angústia e ansiedade nas mães, fazendo com que muitas delas optem pela utilização precoce de alimentos e o uso de mamadeiras, que resulta em práticas inadequadas (MONTESCHIO CAC, et al., 2015).

Os autores ainda relatam que o desmame precoce pode estar relacionado a falta de experiência da mãe, a introdução de chupetas e mamadeiras ou até por ser uma gravidez indesejada. Para Marques ES, et al. (2011) a amamentação só é indicada em casos que as mães são portadoras de HIV, algumas doenças cardíacas, hepáticas graves ou depressão pós-parto além de restrições como o caso de mastites acentuadas ou bebes prematuros.

Diante disso, deixa claro a importância do enfermeiro em fortalecer ações de promoção do aleitamento materno, protegendo e apoiando a prática da amamentação através de uma atuação educacional ampla e contínua. O enfermeiro tem um papel fundamental no AM, visto que são os profissionais que mais se relacionam com a mulher durante o período gravídico puerperal. As práticas em enfermagem oferecem apoio e orientação às gestantes, preparando a mãe para superar eventuais dificuldades, minimizando preocupações e reforçando a autoconfiança. (MARINHO MS, et al., 2015).

Barbieri MC, et al. (2015) destaca que as orientações sobre o AM não se limitam à assistência no pré-natal e na puericultura da atenção básica, e sim se estendem para área hospitalar; pré-parto, parto e puerpério. $O$ êxito no AM depende de vários fatores, dentre eles as recomendações e o suporte dos profissionais da enfermagem que tem como objetivo preparar a mãe para essa experiência. Fortalecendo a autoconfiança e reduzindo as preocupações, quanto melhor a mulher estiver orientada sobre o assunto, maior será a facilidade de superar eventuais obstáculos. 
No período do pré-natal, o enfermeiro deve orientar a gestante sobre as vantagens do aleitamento materno para a mãe, bebê e família; explicar a anatomia e fisiologia das mamas; diferenças na composição do leite materno e outros tipos de leites; consequências do desmame precoce; procedimentos para manter a lactação; alimentação da gestante e nutriz; contracepção e o aleitamento materno; importância do aleitamento em sala de parto e em alojamento conjunto; direitos da mãe e da criança durante o período de aleitamento e a importância de realizar o retorno ao serviço de saúde na primeira semana pós parto (ABRÃO ACFV , 2003).

De acordo com Giugliani CV e Giugliani ERJ (2000) existem estudos que relatam a importância do enfermeiro durante o parto e após, onde os mesmos ajudem as mães nas primeiras mamadas do bebê, estimulando o aleitamento materno após o parto conforme recomendado pela OMS. Carvalho JKM, et al. (2011) chama atenção para que, durante esses trabalhos educativos, o enfermeiro se atente para a assistência da gestante em relação ao preparo das mamas. A gestante deve observar suas mamas todos os dias e realizar exercícios para fortalecer e aumentar a elasticidade do mamilo e da aréola. Há ainda outros cuidados como higiene adequada, não usar sabões, álcool ou qualquer produto secante nos mamilos, além da exposição das mamas ao sol a fim de fortalecê-las. Ainda no alojamento conjunto o enfermeiro deve explicar à mãe as posições para amamentação, prezando sempre pelo conforto da mesma.

Durante a amamentação devem ser colocados o mamilo e o máximo da aréola que for possível, os lábios da criança ficam encurvados para fora, onde ocorre o fechamento entre boca e seio materno. Para interromper a mamada a mãe tem que colocar o dedo mínimo na boca do bebê e o tempo de mamada deve durar o suficiente para sustentar o recém-nascido, que tem que educar após cada mamada.

Logo após o parto, a equipe de enfermagem deverá incentivar e promover a amamentação, devendo ficar junto a mãe para observar a pega do recém-nascido. O AM na primeira hora de vida do recém-nascido, além de reforçar o vínculo mãe bebê, facilita o início da amamentação e protege mulher e criança contra infecções hospitalares. É importante que dentro da maternidade, no alojamento conjunto, sejam reforçadas à mãe as orientações sobre o AM, o cuidado com as mamas e a procurar a unidade de saúde mais perto de sua casa para a continuidade da assistência, tanto da mãe quanto do bebê, durante a puericultura, consulta pós-parto e assistência à nutriz, pela equipe de Saúde da Família (CARVALHO JKM, et al., 2011).

A fase do puerpério é um momento crítico e de mudanças na vida da puérpera, por isso a visita domiciliar do enfermeiro deve ser utilizada para uma consulta de enfermagem, promovendo um atendimento preventivo e resolutivo para os possíveis problemas que possam surgir. $O$ enfermeiro deve explicar à mãe as posições para amamentação, prezando sempre pelo conforto da mesma. Durante a amamentação devem ser colocados o mamilo e o máximo da aréola que for possível na boca do bebê, os lábios da criança ficam encurvados para fora, onde ocorre o fechamento entre boca e seio materno. Para interromper a mamada a mãe tem que colocar o dedo mínimo na boca do bebê e o tempo de mamada deve durar o suficiente para sustentar o recém-nascido, que tem que educar após cada mamada. A consulta de enfermagem ajuda a mãe a entender seus diferentes papéis sociais como o de esposa, o de mãe e o de mulher; além de proporcionar um momento extremamente rico de escuta, vínculo e acolhimento (PEREIRA MC e GRADIM CVC, 2014).

O enfermeiro deve apoiar o momento do retorno da mãe ao trabalho orientando sobre a não introdução de outro tipo de alimento para o bebê, evitando mamadeiras, chupetas. Nesse momento é importante explicar sobre a ordenha das mamas, que é uma alternativa que garante o leite materno e alivia o ingurgitamento mamário entre outros traumas mamilares, e que seja oferecido o leite que foi retirado no copo ou colher (GIUGLIANI CV e GIUGLIANI ERJ, 2000). Um importante meio para que se tenha continuidade do AM é a licença maternidade. De acordo com o Decreto-Lei no 5.452 da Consolidação das Leis de Trabalho de 1943, as mulheres brasileiras que trabalham no mercado formal, tem o direito a 120 dias de licença maternidade remunerada, sem prejuízo do emprego e salário. Quando retornam ao trabalho, elas têm direito a intervalo de meia hora cada turno (manhã e tarde) durante a jornada de trabalho, para que amamente seu bebê até que ele complete seis meses de idade (BRASIL, 1943).

Para a promoção da amamentação é fundamental que o enfermeiro explique para as mães como é a técnica de amamentar e o processo habitual para que frente às intercorrências, elas possam obter êxito na amamentação, reduzindo os sentimentos de ansiedade e insegurança pelo desconhecido (DUCAN BB, 2004).

REAEnf/EJNC | Vol. 2 | e2232 | DOI: https://doi.org/10.25248/REAenf.e2232.2020 Página 5 de 7 


\section{CONSIDERAÇÕES FINAIS}

A prática do AM traz benefícios tanto para a mãe, quanto para o bebê, além de reforçar o vínculo entre eles. O leite materno é alimento rico em nutrientes prevenindo doenças e infecções. Os estudos demonstraram que é importante que o enfermeiro garanta a promoção dessa prática, avaliando constantemente essa faze da mãe, reconhecendo as dificuldades e fortalecendo a autoconfiança. Essas ações podem ser individuais como consultas e/ou visitas domiciliares, mas também através de grupos operativos, como exemplo o grupo de gestante. Porém o comprometimento e responsabilidade do enfermeiro frente ao desmame precoce só é efetivo se outros atores também estiverem envolvidos como a família, sociedade, outros profissionais da saúde e até mesmo o Estado através das políticas públicas.

\section{REFERÊNCIAS}

1. ABRÃO ACFV. Aleitamento materno: seguimentos e controles [resumo]. In: Anias do $2^{\circ}$ Congresso Paulista de Bancos de Leite Humano e 12ํㅡㄹ Encontro Paulista de Aleitamento Materno. Marília, SP, set. 2003; p. 11-14.

2. ALMEIDA JML, et al. Apoio ao aleitamento materno pelos profissionais de saúde: revisão integrativa da literatura. Revista Paulista de Pediatria. 2015. 33(3), 355-362.

3. ALVES EA. Fatores determinantes do desmame precoce: um estudo de revisão bibliográfica. Trabalho de conclusão de curso para obtenção do título de Especialista em Atenção Básica em Saúde da Família. Núcleo de Educação em Saúde Coletiva. Universidade Federal de Minas Gerais. Belo Horizonte, jan. 2010; 49 p.

4. ANDRADE HS, et al. Fatores relacionados ao desmame precoce do aleitamento materno. Revista Brasileira de Medicina de Família e Comunidade. 2018; v. 13, n. 40, p. 1-11.

5. ARAUJO OD, et al. Aleitamento materno: fatores que levam ao desmame precoce. Revista brasileira de enfermagem. 2008, vol.61, n.4, pp.488-492.

6. BARBIERI MC, et al. Aleitamento materno: orientações recebidas no pré-natal, parto e puerpério. Semina: Ciências Biológicas e da Saúde, Londrina, ago. 2015; v. 36, n. 1, supl, p. 17-24.

7. BATISTA KRA, et al. Influência da assistência de enfermagem na prática da amamentação no puerpério imediato. Saúde debate, Rio de Janeiro, mar. 2013; v. 37, n. 96, p. 130-138.

8. BRASIL. Guia Alimentar para Crianças Menores de 2 anos. Ministério da Saúde. Departamento de Atenção Básica. Coordenação Geral de Alimentação e Nutrição. - versão para consulta pública - Brasília, junho/julho de 2018.

9. BRASIL. Presidência da República. Casa Civil. Subchefia para Assuntos Jurídicos. DECRETO-LEI № 5.452, DE 1ํㅡㄹ MAIO DE 1943. Consolidação das Leis do Trabalho.

10. CARVALHO CF e SILVA MGF. Avaliação do desmame precoce e suas implicações infecciosas nas crianças atendidas no ambulatório de um hospital terciário. Arquivo de Ciências da Saúde. São José do Rio Preto, 2005; v. 12 , n. 3, p. $129-32$.

11. CARVALHO JKM, et al. A importância da assistência de enfermagem no aleitamento materno. e- Scientia, Editora UniBH, Belo Horizonte, 2011; v. 4, n. 2, p. 11-20.

12. CARVALHO MJLN, et al. Primeira visita domiciliar puerperal: uma estratégia protetora do aleitamento materno exclusivo. Revista paulista pediatria, São Paulo, mar. 2018; v. 36, n. 1, p. 66-73.

13. CIAMPO LAD, et al. Tendência secular do aleitamento materno em uma unidade de atenção primaria à saúde materno-infantil em Ribeirão Preto, São Paulo. Revista Brasileira de Saúde Materno Infantil. Recife, v.6, n.4, p.391-6.

14. DUNCAN BB. Medicina ambulatorial: Condutas de atenção primária baseadas em evidencias. $3^{\mathrm{a}}$ ed. Porto Alegre: Artmed, 2004.

15. GIUGLIANI CV e GIUGLIANI ERJ. Drogas e Lactação. In: Sociedade Brasileira de Pediatria (org.).PRORN 3módulo 2. Porto Alegre: Artmed/Panamericana, 2000,

16. JOCA MT, et al. Fatores que contribuem para o desmame precoce. Esc. Anna Nery, Rio de Janeiro, Dec. 2005; v. 9, n. 3, p. 356-364.

17. MARINHO MS, et al. A atuação do(a) enfermeiro(a) na promoção, incentivo e apoio ao aleitamento materno: revisão bibliográfica. Revista Enfermagem Contemporânea. Jequié, Jul./Dez 2015; v. 4, n. 2, p. 189-198.

18. MARQUES ES, et al. Mitos e crenças sobre o aleitamento materno. Ciência \& saúde coletiva, Rio de Janeiro, May 2011; v. 16, n. 5, p. 2461-2468. 
19. MONTESCHIO CAC, et al. The nurse faced with early weaning in child nursing consultations. Revista Brasileira de Enfermagem. Cuiabá, 2015; v. 68, n. 5, p. 587-93.

20. MOURA ERBB, et al. Investigação dos fatores sociais que interferem na duração do aleitamento materno exclusivo. Revista Intertox-EcoAdvisor de Toxicologia Risco Ambiental e Sociedade. Picos, jun. 2015; v. 8, n. 2, p. 94-116.

21. NEIVA FCB, et al. Desmame precoce: implicações para o desenvolvimento motor-oral. Jornal de Pediatria. São Paulo, 2003; v.79, n.1.

22. NETO MT. Aleitamento materno e infecção ou da importância do mesmo na sua prevenção. Acta Pediatrica Portuguesa, Lisboa, 2006; v. 37, n. 1, p. 23-26.

23. Obstetrícia, Rio de Janeiro, Dec. 2000; v. 22, n. 10, p. 627-632.

24. OLIVEIRA AK, et al. Práticas e crenças populares associadas ao desmame precoce. Avances em Enfermagem, Bogotá, Dec. 2017; v. 35, n. 3, p. 303-312.

25. PEREIRA MC e GRADIM CVC. Consulta puerperal: a visão do enfermeiro e da puérpera. Ciência, Cuidado e Saúde, Alfenas, Jan/Mar 2014; v. 13, n. 1, p. 35-42.

26. REA MF. Os benefícios da amamentação para a saúde da mulher. Jornal de Pediatria. São Paulo, 2004; v. 80, n. 5(Supl).

27. SALES AN, et al. Mastite Puerperal: Estudo de Fatores Predisponentes. Revista Brasileira de Ginecologia e

28. SANTOS EKA. Aleitamento materno. In: SCHMITZ, EMR.A enfermagem em pediatria e puericultura. São Paulo: Atheneu, 2005. p. 25-48.

29. SILVA DP, et al. Aleitamento materno: causas e consequências do desmame precoce. Revista Unimontes científica, Montes Claros, jul./dez. 2017; v. 19, n.2.

30. SILVA WF e GUEDES ZCF. Tempo de aleitamento materno exclusivo em recém-nascidos prematuros e a termo. Revista CEFAC. São Paulo, 2013; v.15, n.1, p. 160-171.

31. TAMEZ RN e SILVA MJP. Enfermagem Na UTI Neonatal: Assistência Ao Recém-nascido de Alto Risco. 3 ed. Rio de Janeiro: Guanabara Koogan, 2006. p. 170-82.

32. VICTORA CG, et al. Amamentação no século 21: epidemiologia, mecanismos e efeitos ao longo da vida. Epidemiologia e Serviços de Saúde. Pelotas, 2016; p. 1-24. 